\title{
Barotrauma y otras complicaciones frecuentes de la ventilación mecánica en niños
}

\author{
Mario Cerda S. '; Raúl Corrales V. ${ }^{2}$ Enrique Paris M.': Ricardo Ronco M.'
}

\section{Barotrauma in infants and children under mechanical ventilation}

Nonintectious complications in 225 pedialric patients assisted by mechanical veriliation, among 459 patient lages 12 days to 14 years odmitred to o pediatric inilensive care unit along a 32 month period [november 1900 hroughour june 1993! are described. The mast common indicalions for mechanical venlilofion were cardioc surgery in:70|, pneumonia or difuse lung disease in:43\}, septic shock [n:23), meningilis in: 17 ; central nervous

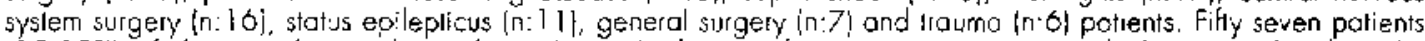
$\{25.33 \%$ of those under mechanical ventilation riad non infectious complicarions, which ware related to the artificial airwoy in 42 (73.3\%) and seconciay to the mechonica! verlilation iself (boratromo and atelectasis) in 15 (26.3\%). The most frequent clirical presentation of barolraumo was pneumothorax [10/14 potienls]. All patients with this compiicalion had been given maximal peak inspiratory pressures obove $40 \mathrm{~cm}$ of waler and the highest averoge values of peok inspiratory pressure ano erd expiratory pressure $156.5 \pm 12.5$ and $11.7 \pm 4.2$ $\mathrm{cm}$ of waler respectivelyl. The whole death rale in this porticulor intensiva core unit wos $9.3 \%$, while among patients under mechonical ventilotion it was $18.2 \%$, including 9 deaths in 14 potients with barotrouma. Becouse $1 / 3$ patients under respiratory ossistance with peak inspiratory pressures above $40 \mathrm{~cm}$ of water hod borolrauma, this presure level shoulo probably nak be exceeded in intants and chi dren.

(Koy words: ventilators mechonical, borolrauma.)

El uso de ventilación mecánica (VM) es una práctica frecuente e indispensable en el manejo de pacientes críticamente enfermos. Sin embargo el procedimiento tiene complicaciones que modifican la evolución de la enfermedad, prolongan la permanencia del paciente en el hospital y pueden poner en riesgo su vida ${ }^{1-3}$.

Las enfermedades por las que se indica VM en pediatría son numerosas, incluyendo infecciones pulmonares y extrapulmonares, malformaciones cardíacas, intoxicaciones, traumatismos, entre orras ${ }^{1.2}$. Los requerimientos ventilatorios en términos de presión inspiratoria máxima (PIM), presión positiva al tinal de la espiración (PEEP), tiempo inspiratorio máxj-

1. Unidad de Tralamiento Intensivo Infantal, Hospital Clínico de la Universidad Católica de Chile.

2. Unidad de Enfermedades Respiratorias Infantiles. Hospital Clínico de la Universidad Católica de Chile, UTl Pediátrica y Laboratorio Broncopulmonar, Clínica Alemana de Santiago. mo (TIM), frecuenca respiratoria (FR) y fracción inspirada de $\mathrm{O}_{2}\left(\mathrm{FiO}_{2}\right.$, varían ampliamente con la edad del paciente y la enfermedad que sufre. Las complicaciones del procedimiento pueden ser secundarias a la vía aérea artificialmente establecida, a la presión positiva empleada 0 a las infecciones secundarias ${ }^{1,2}$.

Se analizan a continuación las complicaciones más frecuentes de la VM en una práctica pediátrica, con excepción de las infecciosas, las características clínicas y las modalidades de apoyo ventilatorio empleadas en los pacientes afectados, con particular énfasis en el barotrauma, con el propósito de contribuir a reducir su frecuencia y consecuencias.

\section{Material y Método}

Mediante un sistema de registro computadorizado prospectivo se analizaron las caractecísticas de los pacientes que ingresaron a la unidad de trataniento intensivo pediátrico del Hospital Clínico de la Pontificia Unjversidad Católica de Chile (UTIP-UC) entre noviembre 
de 1990 a junio de 1993 y requírieron VM. Se registraron las priricipales caracteristicas generales del paciente, la enfermedad por la que se indicó VM. su crolución c intercurrencias. Se consideró edema subglótico postextubación, la obstrucción de la vía aérea superior, que apareció cn las 2 o 3 horas siguientes a la extubación, obligó a reentubar y a emplear iratamiento corticoidal (dexametasona I $\mathrm{mg} \cdot \mathrm{kg}$ en una dosis). La entubación monobronquial se consideró complicación si, habiendo pasado inicialmente inadvertida, determinó deterioro de la condición ventilatoria del paciente. No se consideró complicación la entubación de un bronquio detectada mediante la radiografía de tórax de control al término del procedimiento de instalación del tubo y corregida de inmediato. Las obstruccioncs de lubo endotraqueal se consideraron complicaciones cuando fueron de tal magnitud que interfiriecon en la ventilación y obligaron a cambiarlo. Se definjo como barotrauma la detección, en el examen físico o por rayos $X$, de aire exirapulmonar, ya sea en el intersticio pulmonar. espacio pleural. mediastino o en el celular subcutáneo en la región alta del tórax y cuello. Los neumotórax fueron tratados con Jrenaje pleural, reduciendo a continuación los valores de PIM y PEEP dentro de lo posible, para evitar su reproducción y la absorción del aire desde su ubicación anormal.

El análisis de los datos fue realizado con el programa SAS, en computador Microvax $\$ 400$ del Servicio de Computación de la Escuela de Medicina de la Universidad Católica. Para la comparación entre grupos se utilizó la prueba de Student.

\section{Resultados}

En el periodo del estudio ingresaron a la unidad de cuidados intensivos pediátricos 459 pacientes, $225(49,01 \%)$ requirieron ventilación mecánica y son los sujetos de esta revisión. Sus edades fluctuaron entre 12 días y 14 años $(38,51 \pm 54,32$ meses $): 28$ (21,3\%) eran menores de 2 meses y $51(20,9 \%)$ mayores de 3 años.

Las razones para indicar la ventilación mecánica se describen en la tabla 1, siendo las más frecuentes el postoperatorio de cirugia cardíaca, bronconeumonía, choque séptico y meningoencefalitis. Las presiones aplicadas a la vía aérea y las fracciones inspiradas de oxígeno utilizadas en estos pacientes se resumen en la tabla 2, donde destaca que 42 niños fucron asistidos con presión inspiratoria máxima de 400 más cm de agua, 22 con presión positiva al final de la espiración de 10 o más $\mathrm{cm}$ de agua y 98 con fracciones inspiradas de oxígeno de $80 \%$ o más. La duración de la ventilación mecánica fue menor de 2 días en 90 pacientes, 2 a 7 días en 89 y más de 7 días en 42 pacientes.

\section{Tabla 1}

Causas del empleo de ventilación mecánica en 225 pacientes de la unidad de cuidados intensivos pediátrica

\begin{tabular}{lrr}
\hline Indicación & $\mathbf{n}$ & $\%$ \\
\hline Cirugía cardiaca & 76 & 34,0 \\
Bronconeumonía & 43 & 19,0 \\
Choque séptico & 23 & 10,0 \\
Meningitis & 17 & 8,0 \\
Cirugía cerebral & 16 & 7,0 \\
Estado convulsivo & 11 & 5,0 \\
Insuficiencia cardfaca & 10 & 4,0 \\
Paro cardiorrespiratorio & 8 & 3,5 \\
Cirugía general compleja & 7 & 3,0 \\
Politraumatismo & 6 & 2,6 \\
Otros varios* & 8 & 3,9 \\
\hline Total & 225 & 100,0 \\
\hline
\end{tabular}

* insuficiencia renal: $n=4$; intoxicaciones: $n=2$; insuliciencia hepálica: $\Omega=1$; síndrome de Guillain-Barré: $\mathbf{n}=1$.

Durante el período de estudio se registraron 57 complicaciones, $25,3 \%$ (57/225), según los criterios predefinidos, las que fueron agrupadas en $42(73,7 \%)$ relacionadas con el empleo de una vía aérea artificial y 15 $(26,3 \%)$ secundarias a la aplicación del ventilador mecánico propiamente tal (barotrauma y atelectasia pulmonar masiva). El barotrauma representa casi un cuarto de las complicaciones, afectando a $6,2 \%$ de los pacientes (14/225) sometidos a ventilación mecánica; su forma de presentación más frecuente fue el neumotórax (10/14 casos). Otras complicacioncs fueron neumomediastino ( 3 casos) y enfisema intersticial del pulmón y subcutáneo del cuello en otro caso. De los 14 pacientes con barotrauma 9 fallecieron.

De las variables de aplicación para la ventilación mecánica se revisaron presión inspiratoria máxima, presión positiva del final de la espiración, frecuencia respiratoria, fracción inspirada de oxígeno y duración de la asistencia ventilatoria. Sólo se encontró correlación significativa entre barotrauma y la presión inspiratoria máxima ( $p<0,05$ ), pues todos los 14 casos de barotrauma se registraron entre los 42 pacientes en que esta fue de $40 \mathrm{~cm}$ de $\mathrm{H}_{2} \mathrm{O}$ o más. De] mismo modo las presiones inspira- 
Tabla 2

Ajustes de los factores de ventilación mecánica en 225 pacientes

\begin{tabular}{rcccccccc}
\hline \multicolumn{3}{c}{ PIM $\left(\mathrm{cm}\right.$ de $\left.\mathrm{H}_{2} \mathrm{O}\right)$} & \multicolumn{2}{c}{ PEEP máx $\left(\mathrm{cm}\right.$ de $\left.\mathrm{H}_{2} \mathrm{O}\right)$} & \multicolumn{3}{c}{$F \mathrm{O}_{2}$ máx $(\%)$} \\
\hline$<30$ & $30-<40$ & $>40$ & $<6$ & $6<<10$ & $>10$ & $<50$ & $50-<80$ & $>80$ \\
n: 65 & 118 & 42 & 168 & 35 & 22 & 50 & 77 & 98 \\
\hline
\end{tabular}

n: número de pacientes PIM: presión inspiratoria máxima PEEP: máxima presión positiva de final de espiración $\mathrm{FiO}_{2}$ : fracción inspirada de $\mathrm{O}_{2}$

toria máxima y positiva al final de la espira. ción, así como la fracción de oxígeno en el aire inspirado eran significativamente mayores en los afectados que en los pacientes sin barotrauma (tabla 4). La mortalidad global de los pacientes ingresados a la unidad de cuidados intensivos fue $9,3 \%$, mientras en los sometidos a ventilación mecánica llegó a $18,2 \%(41 / 225)$.

\section{Comentario}

La amplia variedad de la edades y tamaño de los pacientes de esta muestra es caraclerística

Tabla 3

Complicaciones en 225 pacientes tratados con ventilación mecánica

\begin{tabular}{lrr}
\hline Complicaciones & n & $\%$ \\
\hline De la vía aérea artificial: & 18 & 31,6 \\
Edema laríngeo & 7 & 12,3 \\
Intubación de un bronquio & 5 & 8,8 \\
Extubación accidental & 5 & 8,8 \\
Oclusión del tubo traqueal & 1 & 1,7 \\
FV al cambiar tubo traqueal & 1 & 1,7 \\
Rotura de tubo traqueal & 1 & 1,7 \\
$\quad$ Hipercapnia por filtro de aduito & 1 & \\
De la ventilación mećinica: & 14 & 24,6 \\
Barotrauma & 5 & 8,8 \\
Atelectasia masiva & & 100,0 \\
\hline Total & 57 & \\
\hline
\end{tabular}

FV: fíbrilación ventricular de las unidades pediátricas de cuidados intensivos. Las afecciones de los niños que necesitaron ventilación mecánica también lo son ${ }^{1-4}$; en nuestra unidad se agregan, como característica propia, los sometidos a cirugía cardíaca.

El edema laríngeo después de la extubación ocurre casi exclusivamente en niños, por su vía aérea superior estrecha y menos distensible, cuyo diámetro está limitado por el cartílago cricoides, sitio donde es más estrecho ${ }^{2-6}$. El contacto prolongado del tubo endotraqueal con la mucosa subglótica produce edema y disminución del lumen de la vía aérea, que se manifiesta sólo con estridor y obstrucción transitoria durante las primeras 24 horas siguientes a la extubación. Ocasionalmente es necesario tratar al paciente con nebulizaciones de adrenalina racémica y corticoides ev, además de ambiente frío y húmedo. Sin embargo, a veces el edema es tan intenso que puede impedir la extubación: en estos casos, es preciso reentubar con tubos de menor diámetro, tratar con corticoesteroides iv durante 48 horas y reintentar la extubación. La entubación cuidadosa, con tubos adecuados al tamaño del niño y la prohibición de usar implementos vueltos a esterilizar, son recomendaciones básicas para prevenir esta complicación.

La corta longitud de la tráquea del niño favorece la entubación monobronquial. El diámetro interno pequeño, de los tubos traqueales para niños, favorece la obstrucción por secreciones, que puede provocar atelectasias ${ }^{2-6}$. Ambas complicaciones son, por estos motivos, más frecuentes en los niños. La introducción del tubo en un bronquio suele ocurrir durante los intentos de entubación traqueal, pero habitual- 


\section{Tabla 4}

Ajustes máximos de controles ventilatorios en pacientes tratados con ventilación mecánica y su relación con barotrauma (sólo pacientes en quienes se empleó más de $40 \mathrm{~cm}$ de $\mathrm{H}_{2} \mathrm{O}$ de presión inspiratoria máxima

\begin{tabular}{|c|c|c|c|c|c|c|}
\hline & $n$ & $\begin{array}{l}\text { PIM máx } \\
\left(\mathrm{cm} \mathrm{H_{2 }} \mathbf{O} \text { ) }\right.\end{array}$ & $\begin{array}{l}\text { PEEP Máx } \\
\left(\mathrm{cm} \mathrm{H}_{2} \mathrm{O}\right)\end{array}$ & $\begin{array}{c}\text { Duración } \\
\text { de VM (días) }\end{array}$ & $\begin{array}{c}\text { FR máx } \\
\text { (ciclos/min) }\end{array}$ & $\begin{array}{c}\mathrm{FiO}_{\text {, máx }} \\
(\overline{\%})\end{array}$ \\
\hline $\operatorname{con} \mathrm{BT}$ & 14 & $56,5 \pm 12,5$ & $11.7 \pm 4,2$ & $14,4 \pm 19,1$ & $40.1 \pm 8.4$ & $95,0 \pm 3,0$ \\
\hline $\sin B T$ & 28 & $48,9 \pm 6,5^{*}$ & $7, \mathbf{I} \pm 2,9 * *$ & $11,5 \pm 2,5,4$ & $31,7 \pm 9,3$ & $84,0 \pm 2,0 * * *$ \\
\hline
\end{tabular}

Con BT: con barotrauma. sin BT: sin barotrauma. n: número de pacientes

PIM máx: máxima presión inspiratoria máxima aplicada. PEEP máx: ináxima presión positiva de final de espiración. FR máx: máxima frecuencia respiratoria.

$\mathrm{FiO}_{2}$ máx: máxima fracción inspirada de $\mathrm{O}_{2}$

* $p>0,05 \quad$ ** $p<0,001 \quad$ **** $p<0,000$ l. Prueba I de Student.

mente se corrige de inmediato o al comprobar la ubicación inapropiada del tubo en la radiografía de control posterior a su instalación. La entubación inadvertida de un solo bronquio por migración del tubo, con deterioro de la ventilación del paciente, es una complicación grave que requiere tratamiento rápido y puede ser evitada fijando cuidadosamente el tubo una vez comprobada su correcta ubicación.

Los ajustes utilizados en la ventilación mecánica fueron los habituales para las afecciones de los pacientes: unos necesitaron altas presiones inpiratorias máximas para obtener buenos valores de $\mathrm{O}_{2}$ y $\mathrm{CO}_{2}$ en sangre. Algunos necesitaron además presiones positivas altas al final de la espiración para mejorar la oxigenación ${ }^{7}$. Las presiones elevadas se correlacionan significativamente con la incidencia de barotrauma, probablemente debido a aumento de la presion media de la vía aćrea asociado al empleo de volúmenes corrientes elevados.

Es habitual en el manejo de la insuficiencia respiratoria debida a síndrome de dificultad respiratoria aguda (SDRA) y bronconeumonía grave, emplear asistencia ventilatoria con volumen controlado, con el objeto de mantener normal la $\mathrm{PCO}_{2}$, el $\mathrm{pH}$ en 7,40 y tratando de evitar que ocurran atelectasias. Para ello se usan volúmenes corrientes entre 10 y $15 \mathrm{ml} \cdot \mathrm{kg}$ de peso, independientemente de las presiones que con ellos se generen. Recientemente se ha comprobado, en animales y humanos, que este tipo de asistencia de la ventilación se asocia a sobre- distensión alveolar que predispone a rotura del alvéolo y escape de aire hacia el intersticio pulmonar y la cavidad pleural ${ }^{8,9}$. En la génesis de estas alteraciones no parece ser tan importante la presion con que se ventila como el volumen con que se expande el pulmón ${ }^{\dagger 0}$. El barotrauma ya no es definido sólo como un macrobarotrauma (enfísema intersticial neumotórax y neumomediastino), sino también como una lesión que se inicia mucho antes, con la distensión alveolar exagerada, disrupción del epitelio y daño funcional de las células epiteliales y de la via aérea. En otras palabras, el barotrauma no es clínicamente detectable en sus primeras etapas, pero, desde su inicio, causa daño susceptible de apreciar en la función pulmonar (intercambio de gases y distensibilidad) y condiciona la recuperación de ésta y del paciente ${ }^{9-13}$. El barotrauma, en cualesquiera de sus formas, es la complicación más aguda y grave de la ventilación mecánica y requiere tratamiento rápido y efectivo, ya que habitualmente se presenta en pacientes con inestabilidad ventilatoria y hemodinámica. Por estas razones se han buscado otras modalidades de asistencia a la ventilacion, entre las cuales la hipercapnia permisiva parece promisoria. Estudios abiertos no controlados en pacientes con SDRA ${ }^{14-17}$, empleando volúmenes corrientes bajos (entre 6 y $8 \mathrm{ml} \cdot \mathrm{kg}$ ), en que se tolera acumulación de $\mathrm{CO}_{2}$ muy por encima de los valores considerados normales y oxemias o saturaciones bajas (siempre que éstas no se traduzcan en acidosis metabólica, que 
indicaría hipoxia tisular), han mostrado menor frecuencia de barotrauma y mejor pronóstico fínal.

Para evitar el barotrauma es posiblemente preferible ventilar con volúmenes corrientes más pequeños, empleando con cautela las presiones inspiratoric máxima y positiva del final de la espiración. Una costumbre cada vez más frecuente, derivada de lo anterior, es el uso -en estos pacientes- de ventilación limitada por presión, en lugar de volumen.

Otra variable que se relacionó con barotrauma es el empleo de $\mathrm{FiO}_{2}$ elevadas. El empleo simultáneo de PIM. PEEP y FiO, elevados indica más bien la gravedad de la enfermedad pulmonar que motivó la asistencia mecánica de la ventilación que una relación causal entre la $\mathrm{FiO}_{2}$ y el barotrauma. La elevada mortalidad entre los pacientes con barotrauma no obedece a la complicación tanio como a la gravedad de la enfermedad (SDRA o bronconeumonía extensa) por la que fue necesario recurrir a ventilación mecánica ${ }^{18}$.

Otras complicaciones más raras que se relacionan con las características de los pacientes pediátricos (cotura del tubo por mordedura, bipercapnia por gran espacio muerto dcbido al empleo de filtros de humedad para adultos). sólo se ven en niños y el pediatra intensivista debe estar consciente de ellas, ya que no suelen ser descritas en los textos más corrientes sobre aplicaciones de ventiladores mecánicos.

La fibrilación ventricular que ocurrió en uno de nuestros pacientes en rclación al cambio de tubo endotraqueal es de rara incidencia en estas situaciones y puede ser atribuible al uso de succinilcolina como paralizante, ya que mediante una elevación brusca de $\mathrm{K}$ sanguíneo puede provocarla ${ }^{19}$. La hacemos notar por su gravedad, pese a que el paciente -afcctado por sindrome de Guillain-Barré-, resultó indemne de ella a pesar de haber requerido de reanimación cardiopulmonar prolongada (20 min aproximadamente), incluyendo desfibrilación eléctrica en tres oportunidades.

\section{Resumen}

Se describen las complicaciones no infecciosas en niños tratados con asistencia mecánica de la ventilación. Desde noviembre de 1990 a junio de 1993 (32 meses) ingresaron 459 pacientes entre 12 días y 14 años de edad a la unidad de tratamiento intensivo pediátrica del Hospital Clinico de la Universidad Católica, 225 (49,01\%) de los cuales fueron tratados con ventilación mecánica. El motivo del enpleo de respirador mecánico fue cirugia cardíaca en 76 casos, bronconeumonía o enfermedad pulmonar difusa en 43 . choque séptico 23 , meningoencefalitis bacteriana aguda en 17 , cirugía del sistema nervioso central en 16, estado convulsivo prolongado en 11 , cirugía general en 7 y politraumatismo en 6 pacientes. Se registraron 57 complicaciones $(25,33 \%)$ no infecciosas de la ventilación mecánica: edema subglotico postextubación en 18 casos, barotrauma en 14, entubación de un bronquio en 7 , extubación accidental, oclusión de ubo y atelectasias en 5 cada una, fibrilación ventricular al cambiar tubo endotraqueal, hipercapnia por filtro grande y rotura del tubo endotraqueal, todas con un caso. Las complicaciones relacionadas con el uso de vía aérea artificial correspondían a $73,7 \%(42 / 57)$ y las secundarias al emplco de ventilación mecánica (barotrauma y atelectasias) a $26.3 \%(15 / 57)$. La forma de presentación más frecuente del barotrauma, que se registró en 14 casos, fue el neumotórax (10 casos). De los 14 pacientes con barotrauma 9 fallecen. En todos los pacientes que presentaron barotrauma, se usó presiones inspiratorias máximas superiores a $40 \mathrm{~cm}$ de $\mathrm{H}_{2} \mathrm{O}$. Los promedios de presión inspiratoria máxima y presión positiva de línal espiración $\left(56,5 \pm 12,5 \mathrm{~cm} \mathrm{H}_{2} \mathrm{O}\right.$ y $11.7 \pm 4,2 \mathrm{~cm}$ de $\mathrm{H}_{2} \mathrm{O}$ respectivamente) de $\mathrm{los}$ pacientes con barotrauma fueron mayores que en sus iguales sin la complicación. La mortalidad general de la unidad de cuidados intensivos pediátrica en el período del estudio fue $9,3 \%$, ante $18,2 \%$ en los pacientes con ventilación mecánica. Probablemente la presión inspiratoria máxima no debe sobrepasar $40 \mathrm{~cm}$ de $\mathrm{H}_{2} \mathrm{O}$ si se desea evilar el barotrauma.

(Palabras clave: ventilación mecánica, barotrauma).

\section{Referencias}

1. Crone $K$ : Assisted ventilation in children. In: Gegory GA, ed. Respiratory failure in the child, New York. Churchill Livingstone 1981: 17-29.

2. Arriagada S. Cordero J, Baeza J: Complicaciones de la ventilación mecánica en niños. Rev Chil Pediatr 1994: 65: 255-259. 
3. Cerda $M$, Sauvedra $R$, Aspillaga $M$, et at: Trataniento de la insuficiencia respiratoria aguda de origen pulmonar con ventilación mecánica. Rev Chil Pediatr 1984: 55: 25-28.

4. Rivera R, Tibballs $J$ : Complications of cndotracheal intubation and mechanical ventilation in infants and children. Crit Care Med 1992; 20: 193-199.

5. Bery $F$, Yemen $T$ : Pediatric airway in health and disease. Pediatr Clin North Am 1994:41: 15.3-198.

6. Duncan P: Management of upper airway disease in children in: Gregory GA ed. Respiratory failure in the child. New York, Churchill Livingstone 1981: 53-66.

7. Sivan Y, Deakers T. Newth C: Effect of positive endexpriratory pressure on respiratory compliance in children with acute respiratory failure. Pediatr Pulmonol 1991: 1 I: 103-10?.

8. Perkin $R$, Levin $D$ : Adverse effects of posirivepressure ventilation in children. In: Respuratory failure in the child. New York, Churchill Livingstone 1981: $5163 \cdot 187$.

9. Dreyfuss D, Basset G, Soler $P$, Stumon $G$. Intertnittent positive-pressure hyperventilation with high inflation pressure produces microvascular injury in rats. Am Rev Respir Dis 1985; 132: 880-884.

10. Dreyfuss $D$, Soler $P$, Barret $G$, Sammon $G$; High inflation pressure pulmonary edena: respective effects of high airway pressue, high tidal volune. and positive end-expiratory pressure. Am Rev Respir Dis 1988: 137: 1 159-1164

11. Kolbon $T$, Morefri MP, Fumagalli $R$, et al: Severe impairment in lung function induced by high peak airway pressure during mechanical ycntilation. An experimentul study. Am Rev Respir Dis 1987; 135: $312-315$

12. Kawano T. Mori S, Cybulsky $M$, Burger R, el al: Effect of granulocyte depletion in a ventilated surfactant-depleted lung. J Appl Physiol 1987: 62: 27.33 .

13. Parker JC, Townsley $M I$, Rippe B. Taylor AB, et al: Increased microvascular permeability in dog lungs due to high peak airway pressures. J Appl Physiol 1984; 56: 1809-1816.

14. Hickling $K G$ : Low volume ventilation with permissive hypercapria in the adult respiratory distress syndrome. Clinical Intensive Care 1992: 3: 67-68.

15. Reynolds E, Ryan D. Doody D: Permissive hypercapnia and pressure-controlled ventilation as treatment of severe adulu respiratory distress syndrome in a pediatric burn patient. Crit Carc Med 1993: 21 : $944-947$.

16. Kackmorck RM, Hickling KG: Permissive hypercapnia. Resp Care 1993; 38: 373-387.

17. Bidani A. Tzouanakis AE. Cärdenas VJ, Zwischengberger JE: Permissive hypercapnia in adult respiratory failure. JAMA 1994; 272; 957-966.

18. Smith $D$. FrunkeI $L$, Derish $M$, et w: High-frequency jet ventilation in children with the adult respiratory distress syndrome complicated by pulmonary barotrauma. Pediatr Pulmonol 1993: 15: 279-286.

19. Rosenberg H. Gromert G.4: Intractable cardiac arres: in children given succinylcholine. Anesth 1992; 77: 1054-1058.

Esta publicaciốn está disponible en copias de microfilms de 16 y $35 \mathrm{~mm}$ y microfichas de $105 \mathrm{~mm}$, las que pueden solicitarse a:

University Microfilms International

300 North Zeeb Road

Ann Arbor, Michigan 48106, USA.

This journal is also available in $16 \mathrm{~mm}$ microfilm, $35 \mathrm{~mm}$ microfilm and $105 \mathrm{~mm}$ microfilm copies through

University Microfilms International, 300 North Zeeb Road,

Ann Arbor, Michigan 48106, USA. 